\title{
ElimLin Algorithm Revisited
}

\author{
Nicolas T. Courtois ${ }^{1}$, Pouyan Sepehrdad ${ }^{2, \star}$, \\ Petr Sušil ${ }^{2, \star \star}$, and Serge Vaudenay ${ }^{2}$ \\ 1 University College London, UK \\ n. courtois@ucl.ac.uk \\ 2 EPFL, Lausanne, Switzerland \\ \{pouyan.sepehrdad, petr.susil, serge.vaudenay\}@epfl.ch
}

\begin{abstract}
ElimLin is a simple algorithm for solving polynomial systems of multivariate equations over small finite fields. It was initially proposed as a single tool by Courtois to attack DES. It can reveal some hidden linear equations existing in the ideal generated by the system. We report a number of key theorems on ElimLin. Our main result is to characterize ElimLin in terms of a sequence of intersections of vector spaces. It implies that the linear space generated by ElimLin is invariant with respect to any variable ordering during elimination and substitution. This can be seen as surprising given the fact that it eliminates variables. On the contrary, monomial ordering is a crucial factor in Gröbner basis algorithms such as F4. Moreover, we prove that the result of ElimLin is invariant with respect to any affine bijective variable change. Analyzing an overdefined dense system of equations, we argue that to obtain more linear equations in the succeeding iteration in ElimLin some restrictions should be satisfied. Finally, we compare the security of LBlock and MIBS block ciphers with respect to algebraic attacks and propose several attacks on Courtois Toy Cipher version 2 (CTC2) with distinct parameters using ElimLin.
\end{abstract}

Keywords: block ciphers, algebraic cryptanalysis, systems of sparse polynomial equations of low degree.

[Breaking a good cipher should require]

"as much work as solving a system of simultaneous equations in a large number of unknowns of a complex type."

Claude Elwood Shannon 45

\section{Introduction}

Various techniques exist in cryptanalysis of symmetric ciphers. Some involve statistical analysis and some are purely deterministic. One of the latter methods is algebraic attack recognized as early as 1949 by Shannon [45]. Any algebraic attack consists of two distinct stages:

* This work has been supported in part by the European Commission through the ICT program under contract ICT-2007-216646 ECRYPT II.

** Supported by a grant of the Swiss National Science Foundation, 200021_134860/1.

A. Canteaut (Ed.): FSE 2012, LNCS 7549, pp. 306-325, 2012.

(C) International Association for Cryptologic Research 2012 
- Writing the cipher as a system of polynomial equations of low degree often over $\operatorname{GF}(2)$ or $\operatorname{GF}\left(2^{k}\right)$, which is feasible for any cipher [48/20]42].

- Recovering the secret key by solving such a large system of polynomial equations.

Algebraic attacks have been successful in breaking several stream ciphers (see 118/11/24|19|14|23|10 for instance) and a few block ciphers such as Keeloq [37]. and GOST [15, but they are not often as successful as statistical attacks. On the other hand, they often require low data complexity. This is not the case for statistical attacks.

General purpose algebraic attack techniques were developed in the last few years by Courtois, Bard, Meier, Faugère, Raddum, Semaev, Vielhaber, Dinur and Shamir to solve these systems [16/21|20|18/11/30|31/44|47/23/24]. The problem of solving such polynomial systems of multivariate equations is called MQ problem and is known to be NP hard for a random system. Currently, for a random system in which the number of equations is equal to the number of unknowns, there exists no technique faster than an exhaustive key search which can solve such systems. On the other hand, the equations derived from symmetric ciphers turn out to be overdefined and sparse for most ciphers. So, they might be easier to solve. This sparsity is coming from the fact that due to the limitations in hardware and the need for lightweight algorithms, simple operations arise in the definition of cryptosystems. They are also overdefined due to the non-linear operations.

The traditional method for solving overdefined polynomial systems of equations are known to be various Gröbner basis algorithms such as Buchberger algorithm [9, F4 and F5 [30]31] and XL [21]. The most critical drawback of the Gröbner basis approach is the elimination step where the degree of the system increases. This leads to an explosion in memory space and even the most current efficient implementations of Faugère algorithm [30|31] under PolyBoRi framework 8] or Magma [40 are not capable of handling large systems of equations efficiently. On the other hand, they are faster than other methods for overdefined dense systems or when the equations are over $\operatorname{GF}(q)$ where $q>2$. In fact, together with SAT solvers, they are currently the most successful methods for solving polynomial systems.

Nevertheless, due to the technical reasons mentioned above, the system of equations extracted from symmetric ciphers turns out to be sparse. Unfortunately, the Gröbner basis algorithms can not exploit this property. In such cases, algorithms such as XSL [20], SAT solving techniques [4|27/3, Raddum-Semaev algorithm [44] and ElimLin [16] are of interest.

In this paper, we study the elimination algorithm ElimLin that falls within the remit of Gröbner basis algorithms, though it is conceptually much simpler and is based on a mix of simple linear algebra and substitution. It maintains the degree of the equations and it does not require any fixed ordering on the set of all monomials. On the contrary, we need to work with ad-hoc monomial orderings to preserve the sparsity and make it run faster. This simple algorithm reveals 
some hidden linear equations existing in the ideal generated by the system. We show in Sec. 7 that ElimLin does not find all such linear equations.

As far as the authors are aware, no clue has been found yet which demonstrates that ElimLin at some stage stops working. This does not mean that ElimLin can break any system. As mentioned earlier, for a random system this problem is NP hard and Gröbner basis algorithms behave much better for such dense random systems. But, the equations derived from cryptosystems are often not random (see 32 for the huge difference between a random system and the algebraic representation of cryptographic protocols). What we mean here is that if for some small number of rounds ElimLin performs well but then it stops working for more rounds, we can increase the number of samples and it will become effective again. The bottleneck is having an efficient data structure for implementing ElimLin together with a rigorous theory behind it to anticipate its behaviour. These two factors are currently missing in the literature.

Except two simple theorems by Bard (see Chapter 12, Section 5 of [4]), almost nothing has been done regarding the theory behind ElimLin. As ElimLin can also be used as a pre-processing step in any algebraic attack, building a proper theory is vital for improving the state of the art algebraic attacks. We are going to shed some lights on the way this ad-hoc algorithm works and the theory behind it.

In this paper, we show that the output of ElimLin is invariant with respect to any variable ordering. This is a surprising result, i.e., while the spaces generated are different depending on how substitution is performed, we prove that their intersection is exactly the same. Furthermore, we prove that no affine bijective variable change can modify the output of ElimLin. Then, we prove a theorem on how the number of linear equations evolves in each iteration of ElimLin.

An unannounced competition is currently running for designing lightweight cryptographic primitives. This includes several designs which have appeared in the last few years (see [7/22 39|34/29 36 46 2 356]). These designs mainly compete over the gate equivalent (GE) and throughput. This might not be a fair comparison of efficiency, since they do not provide the same level of security with respect to distinct types of attacks. In this paper, we compare the two lightweight Feistel-based block ciphers MIBS [38] and LBlock [49] and show that with the same number of rounds, LBlock provides a much lower level of security compared to MIBS with respect to algebraic attacks. In fact, we attack both ciphers with ElimLin and F4 algorithm. Finally, we provide several algebraic attacks against Courtois Toy Cipher version 2 (CTC2) with distinct parameters using ElimLin.

In Sec. 2, we elaborate the ElimLin algorithm. Then, we remind some basic theorems on ElimLin in Sec. 3. As our main contribution (Theorem 7), we prove in Sec. 4 that ElimLin can be formulated as an intersection of vector spaces. We also discuss its consequences in Sec. 4.2 and prove a theorem regarding the evolution of linear equations in Sec. 4.3. We perform some attacks simulations on CTC2, LBlock and MIBS block ciphers in Sec. 5.2, 5.3 and 5.4 respectively. In Sec. 6, we compare ElimLin and F4. We mention some open problems and a conjecture in Sec. 7 and we conclude. 


\section{ElimLin Algorithm}

ElimLin stands for Eliminate Linear and it is a technique for solving polynomial systems of multivariate equations of low degree $d$ mostly: 2,3 , or 4 over a finite field specifically GF(2). It is also known as "inter-reduction" step in all major algebra systems. As a single tool, it was proposed in [16 to attack DES. It broke 5-round DES. Later, it was applied to break 5-round PRESENT block cipher 43. and to analyze the resistance of Snow 2.0 stream cipher against algebraic attacks [17. It is a simple but a powerful algorithm which can be applied to any symmetric cipher and is capable of breaking their reduced versions. There is no specific requirement for the system except that there should exist at least one linear term, otherwise ElimLin trivially fails. The key question for such an algorithm is to predict its behavior. Currently, very similar to most other types of algebraic attacks such as [47|23|24, multiple parts of the algorithm are heuristic, so it is worthwhile to prove which factors can improve its results, make it run faster or does not have any influence on its ultimate result. This will yield a better understanding of how ElimLin works.

ElimLin is composed of two sequential distinct stages, namely:

- Gaussian Elimination: All the linear equations in the linear span of initial equations are found. They are the intersection between two vector spaces: The vector space spanned by all monomials of degree 1 and the vector space spanned by all equations.

- Substitution: Variables are iteratively eliminated in the whole system based on linear equations until there is no linear equation left. Consequently, the remaining system has fewer variables.

This routine is iterated until no linear equation is obtained in the linear span of the system. See Fig. 1 for a more precise definition of the algorithm. Clearly, the algorithm shall depend on ordering strategies to apply in step 5, 11, and 12 of Fig. 1. We will see that it is not, i.e., the span of the resulting $\mathcal{S}_{L}$ is invariant.

We observe that new linear equations are derived in each iteration of the algorithm that did not exist in the former spans. This phenomenon is called avalanche effect in ElimLin and is the consequence of Theorem 7 . At the end, the system is solved linearly (when $\mathcal{S}_{L}$ is large enough) or ElimLin fails. If the latter occurs, we can increase the data complexity 11 and re-run the attack.

\section{State of the Art Theorems}

The only theoretical analysis of ElimLin was done by Bard in [4. He proved the following theorem and corollary for one iteration of ElimLin:

Theorem 1 ([4]). All linear equations in the linear span of a polynomial equation system $\mathcal{S}^{0}$ are found in the linear span of linear equations derived by performing the first iteration of ElimLin algorithm on the system.

\footnotetext{
${ }^{1}$ For instance, the number of plaintext-ciphertext pairs.
} 
1: Input : A system of polynomial equations $\mathcal{S}^{0}=\left\{\mathrm{Eq}_{1}^{0}, \ldots, \mathrm{Eq}_{m_{0}}^{0}\right\}$ over $\mathrm{GF}(2)$.

2: Output : An updated system of equations $\mathcal{S}^{T}$ and a system of linear equations $\mathcal{S}_{L}$.

3: Set $\mathcal{S}_{L} \leftarrow \emptyset$ and $\mathcal{S}^{T} \leftarrow \mathcal{S}^{0}$ and $k \leftarrow 1$.

4: repeat

5: $\quad$ Perform Gaussian elimination Gauss(.) on $\mathcal{S}^{T}$ with an arbitrary ordering of equations and monomials to eliminate non-linear monomials.

6: $\quad$ Set $\mathcal{S}_{L^{\prime}} \leftarrow$ Linear equations from $\operatorname{Gauss}\left(\mathcal{S}^{T}\right)$.

7: $\quad$ Set $\mathcal{S}^{T} \leftarrow \operatorname{Gauss}\left(\mathcal{S}^{T}\right) \backslash \mathcal{S}_{L^{\prime}}$

8: $\quad$ Set flag.

9: for all $\ell \in \mathcal{S}_{L^{\prime}}$ in an arbitrary order do

10: $\quad$ if $\ell$ is a trivial equation then

11: $\quad$ if $\ell$ is unsolvable then

12: Terminate and output "No Solution".

13: $\quad$ end if

14: else

15: Unset flag.

16: $\quad$ Let $x_{t_{k}}$ be a monomial from $\ell$.

17: $\quad$ Substitute $x_{t_{k}}$ in $\mathcal{S}^{T}$ and $\mathcal{S}_{L}^{\prime}$ using $\ell$.

18: $\quad$ Insert $\ell$ in $\mathcal{S}_{L}$.

19: $\quad k \leftarrow k+1$

20: $\quad$ end if

21: end for

22: until flag is set.

23: Output $\mathcal{S}_{T}$ and $\mathcal{S}_{L}$.

Fig. 1. ElimLin algorithm

The following corollary (also from [4]) is the direct consequence of the above theorem.

Corollary 2. The linear equations generated after performing the first Gaussian elimination in ElimLin algorithm form a basis for all possible linear equations in the linear span of the system.

This shows that any method to perform Gaussian elimination does not affect the linear space obtained at an arbitrary iteration of ElimLin. All linear equations derived from one method exist in the linear span of the equations cumulated from another method. This is trivial to see.

\section{Algebraic Representation of ElimLin}

\subsection{ElimLin as an Intersection of Vector Spaces}

We also formalize ElimLin in an algebraic way. This representation is used in proving Theorem 7 First, we define some notations.

We call an iteration a Gaussian elimination preceding a substitution; The system of equations for ElimLin can be stored as a matrix $\mathcal{M}_{\alpha}$ of dimension 
$m_{\alpha} \times T_{\alpha}$, where each $m_{\alpha}$ rows represents an equation and each $T_{\alpha}$ columns represents a monomial at iteration $\alpha$. Also, $r_{\alpha}$ denotes the rank of $\mathcal{M}_{\alpha}$. Let $n_{\alpha}$ be the number of variables at iteration $\alpha$. We use a reverse lexicographical ordering of columns during Gaussian elimination to accumulate linear equations in the last rows of the matrix. Any arbitrary ordering can be used instead. In fact, we use the same matrix representation as described in [4].

Let $K=\mathrm{GF}(2)$ and $x=\left(x_{1}, \ldots, x_{n}\right)$ be a set of free variables. We denote by $K[x]$ the ring of multivariate polynomials over $K$. For $\mathcal{S} \subset K[x]$, we denote $\operatorname{Span}(\mathcal{S})$ the $K$-vector subspace of $K[x]$ spanned by $\mathcal{S}$. Let $\gamma=\left(\gamma_{1}, \gamma_{2}, \ldots, \gamma_{n}\right)$ be a power vector in $\mathbf{N}^{n}$. The term $x^{\gamma}$ is defined as the product $x^{\gamma}=x_{1}^{\gamma_{1}} \times x_{2}^{\gamma_{2}} \times$ $\cdots \times x_{n}^{\gamma_{n}}$. The total degree of $x^{\gamma}$ is defined as $\operatorname{deg}\left(x^{\gamma}\right) \stackrel{\text { def }}{=} \gamma_{1}+\gamma_{2}+\cdots+\gamma_{n}$. Let Ideal $(\mathcal{S})$ be the ideal spanned by $\mathcal{S}$ and $\operatorname{Root}(\mathcal{S})$ be the set of all tuples $m \in K^{n}$ such that $f(m)=0$ for all $f \in \mathcal{S}$. Let

$$
\left.R_{d}=\text { Span (monomials of degree } \leq d\right) / \text { Ideal }\left(x_{1}^{2}-x_{1}, x_{2}^{2}-x_{2}, \ldots, x_{n}^{2}-x_{n}\right)
$$

Let $\mathcal{S}^{\alpha}$ be $\mathcal{S}^{T}$ after the $\alpha$-th iteration of ElimLin and $\mathcal{S}^{0}$ be the initial system. Moreover, $n_{L}^{\alpha}$ is the number of non-trivial linear equations in $\mathcal{S}_{L^{\prime}}$ at the $\alpha$-th iteration. We denote $\mathcal{S}_{L}^{\alpha}$ the $\mathcal{S}_{L}$ after the $\alpha$-th iteration. Also, $C^{\alpha} \stackrel{\text { def }}{=} \# \mathcal{S}_{L}^{\alpha}$.

Let assume that $\mathcal{S}^{0}$ has degree bounded by $d$. We denote by $\operatorname{Var}(f)$ the set of variables $x_{i}$ expressed in $f$. Let $x_{t_{1}}, \ldots, x_{t_{k}}$ be the sequence of eliminated variables. We define $\mathrm{V}_{k}=\left\{x_{1}, \ldots, x_{n}\right\} \backslash\left\{x_{t_{1}}, \ldots, x_{t_{k}}\right\}$. Also, let $\ell_{1}, \ell_{2}, \ldots, \ell_{k}$ be the sequence of linear equations as they are used during elimination (step 11 of Fig. 11). Hence, we have $x_{t_{k}} \in \operatorname{Var}\left(\ell_{k}\right) \subseteq \mathrm{V}_{k-1}$.

We prove the following crucial lemma which we use later to prove Theorem 7 .

Lemma 3. After the $\alpha$-th iteration of ElimLin, an arbitrary equation $\mathrm{Eq}_{i}^{\alpha}$ in the system $\left(\mathcal{S}^{\alpha} \cup \mathcal{S}_{L}^{\alpha}\right)$ for an arbitrary $i$ can be represented as

$$
\mathrm{Eq}_{i}^{\alpha}=\sum_{t=1}^{m_{0}} \beta_{t i}^{\alpha} \cdot \mathrm{Eq}_{t}^{0}+\sum_{t=1}^{C^{\alpha}} \ell_{t}(x) \cdot g_{t i}^{\alpha}(x)
$$

where $\beta_{t i}^{\alpha} \in K$ and $g_{t i}^{\alpha}(x)$ is a polynomial in $R_{d-1}$ and $\operatorname{Var}\left(g_{t i}^{\alpha}\right) \subseteq \mathrm{V}_{t}$.

Proof. Let $x_{t_{1}}$ be one of the monomials existing in the first linear equation $\ell_{1}(x)$ and this specific variable is going to be eliminated. Substituting $x_{t_{1}}$ in an equation $x_{t_{1}} \cdot h(x)+z(x)$, where $h(x)$ has degree at most $d-1, x_{t_{1}} \notin \operatorname{Var}(h)$ and $x_{t_{1}} \notin \operatorname{Var}(z)$ is identical to subtracting $h(x) \cdot \ell_{1}(x)$. Consequently, the proof follows by induction on $\alpha$.

Now, we prove the inverse of the above lemma.

Lemma 4. For each $i$ and each $\alpha$, there exists $\beta_{t i}^{\prime \alpha} \in K$ and $g_{t i}^{\prime \alpha}(x)$ such that

$$
\mathrm{Eq}_{i}^{0}=\sum_{t=1}^{m_{\alpha}} \beta_{t i}^{\prime \alpha} \cdot \mathrm{Eq}_{t}^{\alpha}+\sum_{t=1}^{C^{\alpha}} \ell_{t}(x) \cdot g_{t i}^{\prime \alpha}(x)
$$

where $g_{t i}^{\prime \alpha}(x)$ is a polynomial in $R_{d-1}$ and $\operatorname{Var}\left(g_{t i}^{\prime \alpha}\right) \subseteq \mathrm{V}_{t}$. 
Proof. Gaussian elimination and substitution are invertible operations. We can use a similar induction as the previous lemma to prove the above equation.

In the next lemma, we prove that $\mathcal{S}_{L}^{\alpha}$ contains all linear equations which can be written in the form of Eq. (11).

Lemma 5. If there exists $\ell \in R_{1}$ and some $\beta_{t}$ and $g_{t}^{\prime \prime}(x)$ such that

$$
\ell(x)=\sum_{t=1}^{m_{0}} \beta_{t} \cdot \mathrm{Eq}_{t}^{0}+\sum_{t=1}^{C^{\alpha}} \ell_{t}(x) \cdot g_{t}^{\prime \prime}(x)
$$

at iteration $\alpha$, where $g_{t}^{\prime \prime}(x)$ is a polynomial in $R_{d-1}$, then there exists $u_{t} \in K$ and $v_{t} \in K$ such that

$$
\ell(x)+\sum_{t=1}^{C^{\alpha}} u_{t} \cdot \ell_{t}(x)=\sum_{t=1}^{m_{\alpha}} v_{t} \cdot \mathrm{Eq}_{t}^{\alpha}
$$

So, $\ell(x) \in \operatorname{Span}\left(\mathcal{S}_{L}^{\alpha}\right)$.

Proof. We define $u_{k}$ iteratively: $u_{k}$ is the coefficient of $x_{t_{k}}$ in

$$
\ell(x)+\sum_{t=1}^{k-1} u_{t} \cdot \ell_{t}(x)
$$

for $k=1, \ldots, C^{\alpha}$. So, $\operatorname{Var}\left(\ell(x)+\sum_{t=1}^{k} u_{t} \cdot \ell_{t}(x)\right) \subseteq \mathrm{V}_{k}$. By substituting $\mathrm{Eq}_{i}^{0}$ from Eq. (2) in Eq. (3) and integrating $u_{t}$ and $g_{t}^{\prime \prime}$ in $g_{t i}^{\prime \alpha}$, we obtain

$$
\underbrace{\ell(x)+\sum_{t=1}^{C^{\alpha}} u_{t} \cdot \ell_{t}(x)}_{\subseteq \mathrm{V}_{1}}=\underbrace{\sum_{t=1}^{m_{\alpha}} v_{t} \cdot \mathrm{Eq}_{t}^{\alpha}}_{\subseteq \mathrm{V}_{1}}+\underbrace{\sum_{t=1}^{C^{\alpha}} \ell_{t}(x) \cdot g_{t}^{\prime}(x)}_{\Longrightarrow \subseteq \mathrm{V}_{1}}
$$

with $g_{t}^{\prime}(x) \in R_{d-1}$. All $g_{t}^{\prime}(x)$ where $t>1$ can be written as $\bar{g}_{t}(x)+x_{t_{1}} \cdot \overline{\bar{g}}_{t}(x)$ with $\operatorname{Var}\left(\bar{g}_{t}\right) \subseteq \mathrm{V}_{1}, \operatorname{Var}\left(\overline{\bar{g}}_{t}\right) \subseteq \mathrm{V}_{1}$ and $\overline{\bar{g}}_{t}(x) \in R_{d-2}$. Since,

$$
\begin{aligned}
\ell_{1}(x) \cdot g_{1}^{\prime}(x)+\ell_{t}(x) \cdot g_{t}^{\prime}(x)= & \ell_{1}(x) \cdot \underbrace{\left(g_{1}^{\prime}(x)+\ell_{t}(x) \cdot \overline{\bar{g}}_{t}(x)\right)}_{\text {new } g_{1}^{\prime}(x)} \\
& +\underbrace{\ell_{t}(x)}_{\subseteq \mathrm{V}_{1}} \cdot \underbrace{\left(\bar{g}_{t}(x)+\overline{\bar{g}}_{t}(x) \cdot\left(x_{t_{1}}-\ell_{1}(x)\right)\right)}_{\left(\text {new } g_{t}^{\prime}(x)\right) \subseteq \mathrm{V}_{1}}
\end{aligned}
$$

we can re-arrange the sum in Eq. (4) using the above representation and obtain $\operatorname{Var}\left(g_{t}^{\prime}\right) \subseteq \mathrm{V}_{1}$ for all $t>1$. Also, $x_{t_{1}}$ only appears in $\ell_{1}(x)$ and $g_{1}^{\prime}(x)$. So, the coefficient of $x_{t_{1}}$ in the expansion of $\ell_{1}(x) \cdot g_{1}^{\prime}(x)$ must be zero. In fact, we have

$$
\begin{aligned}
\ell_{1}(x) \cdot g_{1}^{\prime}(x) & =\left(x_{t_{1}}+\left(\ell_{1}(x)-x_{t_{1}}\right)\right) \cdot\left(\bar{g}_{1}(x)+x_{t_{1}} \cdot \overline{\bar{g}}_{t}(x)\right) \\
& =x_{t_{1}} \cdot\left(\overline{\bar{g}}_{1}(x) \cdot\left(1+\ell_{1}(x)-x_{t_{1}}\right)+\bar{g}_{1}(x)\right)+\bar{g}_{1}(x) \cdot\left(\ell_{1}(x)-x_{t_{1}}\right)
\end{aligned}
$$


So, $\bar{g}_{1}(x)=\overline{\bar{g}}_{1}(x) \cdot\left(x_{t_{1}}-\ell_{1}(x)-1\right)$ and we deduce,

$$
g_{1}^{\prime}(x)=\overline{\bar{g}}_{1}(x) \cdot\left(\ell_{1}(x)+1\right)
$$

over $\mathrm{GF}(2)$. But, then $\ell_{1}(x) \cdot g_{1}^{\prime}(x)=0$ over $R$, since $\ell_{1}(x) \cdot\left(\ell_{1}(x)+1\right)=0$. Finally, we iterate and obtain

$$
\ell(x)+\sum_{t=1}^{C^{\alpha}} u_{t} \cdot \ell_{t}(x)=\sum_{t=1}^{m_{\alpha}} v_{t} \cdot \mathrm{Eq}_{t}^{\alpha}
$$

From another perspective, ElimLin algorithm can be represented as in Fig. 2. In fact, as a consequence of Lemma 3 and Lemma [5. Fig. 2 presents a unique characterization of $\operatorname{Span}\left(\mathcal{S}_{L}\right)$ in terms of a fixed point:

1: Input : A set $\mathcal{S}^{0}$ of polynomial equations in $R_{d}$.

2: Output : A system of linear equations $\mathcal{S}_{L}$.

3: Set $\overline{\mathcal{S}_{L}}:=\emptyset$.

4: repeat

5: $\quad \overline{\mathcal{S}}_{L} \leftarrow \operatorname{Span}\left(\mathcal{S}^{0} \cup\left(R_{d-1} \times \overline{\mathcal{S}}_{L}\right)\right) \cap R_{1}$

6: until $\overline{\mathcal{S}_{L}}$ unchanged

7: Output $\mathcal{S}_{L}$ : a basis of $\overline{\mathcal{S}}_{L}$.

Fig. 2. ElimLin algorithm from another perspective

Lemma 6. At the end of ElimLin, Span $\left(\mathcal{S}_{L}\right)$ is the smallest subset $\overline{\mathcal{S}}_{L}$ of $R_{1}$, such that

$$
\overline{\mathcal{S}}_{L}=\operatorname{Span}\left(\mathcal{S}^{0} \cup\left(R_{d-1} \times \overline{\mathcal{S}}_{L}\right)\right) \cap R_{1}
$$

Proof. By induction, at step $\alpha$ we have $\overline{\mathcal{S}}_{L} \subseteq$ Span $\left(\mathcal{S}_{L}^{\alpha}\right)$, using Lemma 5. Also, $\mathcal{S}_{L}^{\alpha} \subseteq \overline{\mathcal{S}}_{L}$ using Lemma 3. So, $\overline{\mathcal{S}}_{L}=\operatorname{Span}\left(\mathcal{S}_{L}^{\alpha}\right)$ at step $\alpha$. Since

$$
\overline{\mathcal{S}}_{L} \mapsto \operatorname{Span}\left(\mathcal{S}^{0} \cup\left(R_{d-1} \times \overline{\mathcal{S}}_{L}\right)\right) \cap R_{1}
$$

is increasing, we obtain the above equation.

ElimLin eliminates variables, thus it looks very unexpected that the number of linear equations in each step of the algorithm is invariant with respect to any variable ordering in the substitution step and the Gaussian elimination. We finally prove this important invariant property. Concretely, we formalize ElimLin as a sequence of intersection of vector spaces. Such intersection in each iteration is between the vector space spanned by the equations and the vector space generated by all monomials of degree 1 in the system. This implies that if ElimLin runs for $\alpha$ iterations (finally succeeds or fails), it can be formalized as a sequence of intersections of $\alpha$ pairs of vector spaces. These intersections of vector spaces only depend on the vector space of the initial system. 
Theorem 7. The following relations exist after running ElimLin on a polynomial system of equations $Q$ :

1. $\operatorname{Root}\left(\mathcal{S}^{0}\right)=\operatorname{Root}\left(\mathcal{S}^{T} \cup \mathcal{S}_{L}\right)$

2. There is no linear equation in $\operatorname{Span}\left(\mathcal{S}^{T}\right)$.

3. $\operatorname{Span}\left(\mathcal{S}_{L}\right)$ is uniquely defined by $\mathcal{S}^{0}$.

4. $\mathcal{S}_{L}$ consists of linearly independent linear equations.

5. The complexity is $O\left(n_{0}^{d+1} m_{0}^{2}\right)$, where $d$ is the degree of the system and $n_{0}$ and $m_{0}$ are the initial number of variables and equations, respectively.

Proof (1). Due to Lemma 3 and Lemma 4, $\mathcal{S}^{0}$ and $\left(\mathcal{S}^{T} \cup \mathcal{S}_{L}\right)$ are equivalent. So, a solution of $\mathcal{S}^{0}$ is also a solution of $\left(\mathcal{S}^{T} \cup \mathcal{S}_{L}\right)$ and vice versa.

Proof (2). Since ElimLin stops on $\mathcal{S}^{T}$, the Gaussian reduction did not find any linear polynomial.

Proof (3). Due to Lemma 6.

Proof (4). $\mathcal{S}_{L}$ includes a basis for $\overline{\mathcal{S}}_{L}$. So, it consists of linearly independent equations.

Proof (5). $n_{0}$ is an upper bound on $\# \mathcal{S}_{L}$ due to the fact that $\mathcal{S}_{L}$ consists of linearly independent linear equations. So, the number of iterations is bounded by $n_{0}$. The total number of monomials is bounded by

$$
T_{0} \leq \sum_{i=0}^{d}\left(\begin{array}{c}
n_{0} \\
i
\end{array}\right)=O\left(n_{0}^{d}\right)
$$

The complexity of Gaussian elimination is $O\left(m_{0}^{2} T_{0}\right)$, since we have $T_{0}$ columns and $m_{0}$ equations. Therefore, overall, the complexity of ElimLin is $O\left(n_{0}^{d+1} m_{0}^{2}\right)$.

\subsection{Affine Bijective Variable Change}

In the next theorem, we prove that the result of ElimLin algorithm does not change for any affine bijective variable change. It is an open problem to find an appropriate non-linear variable change which improves the result of the ElimLin algorithm.

Theorem 8. Any affine bijective variable change $A: \mathrm{GF}(2)^{n_{0}} \rightarrow \mathrm{GF}(2)^{n_{0}}$ on a $n_{0}$-variable system of equations $\mathcal{S}^{0}$ does not affect the result of ElimLin algorithm, implying that the number of linear equations generated at each iteration is invariant with respect to an affine bijective variable change.

Proof. In Lemma 6, we showed that Span $\left(\mathcal{S}_{L}\right)$ is the output of the algorithm in Fig. 2, iterating

$$
\overline{\mathcal{S}}_{L} \leftarrow \operatorname{Span}\left(\mathcal{S}^{0} \cup\left(R_{d-1} \times \overline{\mathcal{S}}_{L}\right)\right) \cap R_{1}
$$

We represent the composition of a polynomial $f_{1}$ with respect to $A$ by $\operatorname{Com}\left(f_{1}\right)$.

We then show that there is a commutative diagram. 


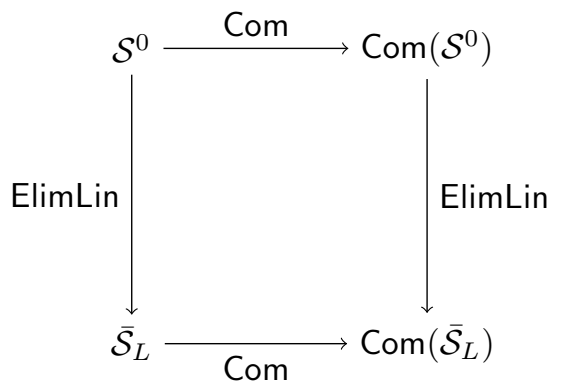

We consider two parallel executions of the algorithm in Fig. 2, one with $\mathcal{S}^{0}$ and the other with $\operatorname{Com}\left(\mathcal{S}^{0}\right)$.

If we compose the polynomials in $\mathcal{S}^{0}$ with respect to $A$, in the above relation $R_{d-1}$ remains the same. Since the transformation $A$ is affine,

$\operatorname{Com}\left(\operatorname{Span}\left(\mathcal{S}^{0} \cup\left(R_{d-1} \times \overline{\mathcal{S}}_{L}\right)\right) \cap R_{1}\right)=\operatorname{Span}\left(\operatorname{Com}\left(\mathcal{S}^{0}\right) \cup\left(R_{d-1} \times \operatorname{Com}\left(\overline{\mathcal{S}}_{L}\right)\right)\right) \cap R_{1}$

So, at each iteration, the second execution has the result of applying Com to the result of the first one.

\subsection{Linear Equations Evolution}

An open problem regarding ElimLin is to predict how the number of linear equations evolves in the preceding iterations. In the following theorem, we give a necessary (but not sufficient) condition for a dense overdefined system of equations to have an additional linear equation in the next iteration of ElimLin. Proving a similar result for a sparse system is not straightforward.

Theorem 9. If we apply ElimLin to an overdefined dense system of quadratic equations over $\mathrm{GF}(2)$, for $n_{L}^{\alpha+1}>n_{L}^{\alpha}$ to hold, it is necessary to have

$$
\frac{b_{\alpha}}{2}-a_{\alpha}<n_{L}^{\alpha}<\frac{b_{\alpha}}{2}+a_{\alpha}
$$

where $b_{\alpha}=2 n_{\alpha}-1$ and $a_{\alpha}=\frac{\sqrt{b_{\alpha}^{2}-8 n_{L}^{\alpha}}}{2}$.

Proof. For the system to generate linear equations, it is necessary that the sufficient rank condition [4] is satisfied. More clearly, we must have $r_{\alpha}>T_{\alpha}-1-n_{\alpha}$, otherwise no linear equations will be generated. This is true if the system of equations is overdefined. Hence, we obtain,

$$
n_{L}^{\alpha}=r_{\alpha}+n_{\alpha}+1-T_{\alpha}
$$

If some columns of the matrix $\mathcal{M}_{\alpha}$ are pivotless, it will shift the diagonal strand of ones to the right. Therefore, $n_{L}^{\alpha}$ will be more than what the above equation expresses. Assuming the system of equations is dense, this phenomenon happens with a very low probability. Suppose the above equation is true with high probability, then we get

$$
n_{L}^{\alpha+1}=r_{\alpha+1}+n_{\alpha+1}+1-T_{\alpha+1}
$$


In the $(\alpha+1)$-th iteration, the number of variables is reduced by $n_{L}^{\alpha}$. Thus, $n_{\alpha+1}=n_{\alpha}-n_{L}^{\alpha}$. If the system of equations is dense, in a quadratic system,

$$
T_{\alpha}=\left(\begin{array}{c}
n_{\alpha} \\
2
\end{array}\right)+n_{\alpha}+1
$$

and so,

$$
T_{\alpha+1}=\left(\begin{array}{c}
n_{\alpha}-n_{L}^{\alpha} \\
2
\end{array}\right)+n_{\alpha}-n_{L}^{\alpha}+1
$$

Consequently, we have

$$
T_{\alpha}-T_{\alpha+1}=n_{L}^{\alpha}\left(n_{\alpha}-\frac{1}{2}\left(n_{L}^{\alpha}-1\right)\right)
$$

Therefore, using Eq. (5), Eq. (6) and Eq. (7), we obtain,

$$
\begin{aligned}
n_{L}^{\alpha+1} & =\left(r_{\alpha+1}-r_{\alpha}\right)+\left(r_{\alpha}+n_{\alpha}-T_{\alpha}+1\right)+n_{L}^{\alpha}\left(-\frac{1}{2} n_{L}^{\alpha}+n_{\alpha}-\frac{1}{2}\right) \\
& =n_{L}^{\alpha}\left(-\frac{1}{2} n_{L}^{\alpha}+n_{\alpha}+\frac{1}{2}\right)-\left(r_{\alpha}-r_{\alpha+1}\right)
\end{aligned}
$$

If $n_{L}^{\alpha+1}>n_{L}^{\alpha}$, then $n_{L}^{\alpha}\left(-\frac{1}{2} n_{L}^{\alpha}+n_{\alpha}+\frac{1}{2}\right)-\left(r_{\alpha}-r_{\alpha+1}\right)>n_{L}^{\alpha}$ and this leads to

$$
n_{L}^{\alpha 2}+\left(1-2 n_{\alpha}\right) n_{L}^{\alpha}+2\left(r_{\alpha}-r_{\alpha+1}\right)<0
$$

$\Delta=\left(1-2 n_{\alpha}\right)^{2}-8\left(r_{\alpha}-r_{\alpha+1}\right)$, and if the above inequality holds, $\Delta$ should be positive and assuming $b_{\alpha}=2 n_{\alpha}-1$, then, $b_{\alpha}-\sqrt{\Delta}<2 n_{L}^{\alpha}<b_{\alpha}+\sqrt{\Delta}$.

Considering $\Delta$ is positive, $n_{\alpha}>\sqrt{2\left(r_{\alpha}-r_{\alpha+1}\right)}+\frac{1}{2}$. We also know that $r_{\alpha+1} \leq$ $r_{\alpha}-n_{L}^{\alpha}$, which together lead to $n_{\alpha}>\frac{1}{2}+\sqrt{2 n_{L}^{\alpha}}$. Therefore, for $n_{L}^{\alpha+1}>n_{L}^{\alpha}$, it is necessary to have $n_{\alpha}>\frac{1}{2}+\sqrt{2 n_{L}^{\alpha}}$, but not visa versa. Simplifying $b_{\alpha}-\sqrt{\Delta}<$ $2 n_{L}^{\alpha}<b_{\alpha}+\sqrt{\Delta}$ and deploying $r_{\alpha}-r_{\alpha+1} \geq n_{L}^{\alpha}$ results in

$$
b_{\alpha}-2 a_{\alpha}<2 n_{L}^{\alpha}<b_{\alpha}+2 a_{\alpha}
$$

where $b_{\alpha}=2 n_{\alpha}-1$ and $2 a_{\alpha}=\sqrt{b_{\alpha}^{2}-8 n_{L}^{\alpha}}$.

Notice that $n_{\alpha}>\frac{1}{2}+\sqrt{2 n_{L}^{\alpha}}$, which was obtained in the first stage of the proof, has been originated from the fact that $b_{\alpha}^{2}-8 n_{L}^{\alpha}$ should be non-negative.

\section{Attacks Simulations}

In this section, we present our experimental results against CTC2, LBlock and MIBS block ciphers. The simulations for CTC2 were run on an ordinary PC with a $1.8 \mathrm{Ghz}$ CPU and $2 \mathrm{~GB}$ RAM. All the other simulations were run on an ordinary PC with a $2.8 \mathrm{Ghz} \mathrm{CPU}$ and $4 \mathrm{~GB}$ RAM. The amount of RAM required by our implementation is negligible. 
In our attacks, we build a system of quadratic equations with variables representing plaintext, ciphertext, key and state bits, which allows to express the system of equations of high degree as quadratic equations. Afterwards, for each sample we set the plaintext and ciphertext according to the result of the input/output of the cipher. In order to test the efficiency of the algebraic attack, we guess some bits of the key and set the key variables corresponding to the guess. Then, we run the solver (ElimLin, F4 or SAT solver) to recover the remaining key bits and test whether the guess was correct. Therefore, the complexity of our algebraic attack can be bounded by $2^{g} \cdot \mathcal{C}($ solver $)$, where $\mathcal{C}($ solver $)$ represents the running time of the solver and $g$ is the number of bits we guess. $\mathcal{C}($ solver $)$ is represented as the the "Running Time" in all the following tables.

For a comparison with a brute force attack, we consider a fair implementation of the cipher, which requires $10 \mathrm{CPU}$ cycles per round. This implies that the algebraic attack against $t$ rounds of the cipher is faster than an exhaustive search for the $1.8 \mathrm{Ghz}$ and $2.8 \mathrm{Ghz} \mathrm{CPU}$ iff recovering $c$ bits of the key is faster than $5.55 \cdot t \cdot 2^{c-31}$ and $3.57 \cdot t \cdot 2^{c-31}$ seconds respectively. This is already twice faster than the complexity of exhaustive search. All the attacks reported in the following tables are faster than exhaustive search with the former argument. In fact, we consider the cipher to be broken for some number of rounds if the algebraic attack that recovers $(\# k e y-g)$ key bits is faster than an exhaustive key search over $(\# k e y-g)$ bits of the key.

\subsection{Simulations Using F4 Algorithm under PolyBoRi Framework}

The most efficient implementation of the F4 algorithm is available under PolyBoRi framework [8] running alone or under SAGE algebra system. PolyBoRi is a $\mathrm{C}++$ library designed to compute Gröbner basis of an ideal applied to Boolean polynomials. A Python interface is used, surrounding the $\mathrm{C}++$ core. It uses zero-suppressed binary decision diagrams (ZDDs) 33] as a high level data structure for storing Boolean polynomials. This representation stores the monomials more efficiently in memory and it makes the Gröbner basis computation faster compared to other algebra systems.

We use polybori-0.8.0 for our attacks. Together with ElimLin, we also attack LBlock and MIBS with F4 algorithm and then compare PolyBoRi's efficiency with our implementation of ElimLin.

\subsection{Simulations on CTC2}

Courtois Toy Cipher (CTC) is an SPN-based block cipher devised by Courtois [13. as a toy cipher to evaluate algebraic attacks on smaller variants of cryptosystems. It was designed to show that it is possible to break a cipher using an ordinary PC deploying a small number of known or chosen plaintext-ciphertext pairs. 
Since the system of equations of well-known ciphers such as AES is often large, it is not feasible by the current algorithms and computer capacities to solve them in a reasonable time, therefore smaller but similar versions such as CTC can be exploited to evaluate the resistance of ciphers against algebraic cryptanalysis. This turns out to yield a benchmark on understanding the algebraic structure of ciphers. Ultimately, this might lead to break of a larger system later.

CTC was not designed to be resistant against all known types of attacks like linear and differential cryptanalysis. Nevertheless, in [25], it was attacked by linear cryptanalysis. Subsequently, CTC Version 2 or CTC2 was proposed [12 to resolve the flaw exists in CTC structure. CTC2 is very similar to CTC with a few changes. It is an SPN-based network with scalable number of rounds, block and key size. For the full specification, refer to [12]. In CT-RSA 2009, differential and differential-linear attacks could reach up to 8 rounds of CTC2 [26], but as stated before, the objective of the CTC designer was not applying statistical attacks to his design. Finally, there is a cube attack on 4 rounds of one variant of this cipher in 41 .

Since block size and key size are flexible in CTC2 cipher, we break various versions with distinct parameters (see Table 1) using ElimLin. The block size is specified by a parameter $B$, which specifies the number of parallel S-boxes per round. CTC2 S-box is $3 \times 3$, hence the block size is computed as $3 B$. We guess some LSB bits of the key and we show that recovering the remaining is faster than exhaustive search.

It might be possible that during the intermediate steps of ElimLin, a quadratic equation in only key bits (possibly linear) appears. In such cases, approximately $\mathcal{O}\left(\# k e y^{2}\right)$ samples are enough to break the system. This is due to the fact that we can simply change the plaintext-ciphertext pair and generate a new linearly independent equation in the key. Finally, when we have enough such equations, we solve a system of quadratic equations in only key bits using the linearization technique. When such phenomenon occurs, intuitively the cipher is close to be broken but not yet. We can increase the number of samples and most often it makes the cipher thoroughly collapse.

\subsection{Simulations on LBlock}

LBlock is a new lightweight Feistel-based block cipher, aimed at constrained environments, such as RFID tags and sensor networks 49. proposed at ACNS 2011. It operates on 64-bit blocks, uses a key of 80 bits and iterates 32 rounds. For a detailed specification of the cipher, refer to [49]. As far as the authors are aware, there is currently no cryptanalysis results published on this cipher.

We break 8 rounds of LBlock using 6 samples deploying an ordinary PC by ElimLin. Our results are summarized in Table 2. In the same scenario, PolyBoRi crashes due to running out of memory. 
Table 1. CTC2 simulations using ElimLin up to 6 rounds with distinct parameters

\begin{tabular}{|c|c|c|c|c|c|c|c|}
\hline$B$ & $\overline{N_{r}}$ & \#key & $g$ & $\begin{array}{l}\text { Running Time } \\
\text { (in hours) }\end{array}$ & $\begin{array}{l}\text { Running Time } \\
\text { (in hours) }\end{array}$ & Data & $\begin{array}{c}\text { Attack } \\
\text { notes }\end{array}$ \\
\hline 16 & 3 & 48 & 0 & \multirow[t]{4}{*}{0.03} & & $\begin{array}{ll}5 \mathrm{KP} \\
\end{array}$ & ElimLin \\
\hline 16 & 3 & 48 & 0 & & 0.12 & $14 \mathrm{KP}$ & ElimLin \\
\hline 64 & 3 & 192 & 155 & & 0.03 & $1 \mathrm{KP}$ & ElimLin \\
\hline 85 & 3 & 255 & 210 & & 0.04 & $1 \mathrm{KP}$ & ElimLin \\
\hline 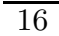 & 4 & 48 & 0 & \multirow[t]{2}{*}{0.01} & \multirow{3}{*}{0.05} & $\begin{array}{|ll|}2 & \mathrm{CP} \\
\end{array}$ & \begin{tabular}{|l|} 
ElimLin \\
\end{tabular} \\
\hline 16 & 4 & 48 & 0 & & & $4 \mathrm{CP}$ & ElimLin \\
\hline 40 & 4 & 120 & 85 & \multirow[t]{3}{*}{0.00} & & $1 \mathrm{KP}$ & ElimLin \\
\hline 40 & 4 & 120 & 85 & & 0.84 & $16 \mathrm{KP}$ & ElimLin \\
\hline 48 & 4 & 144 & 100 & & 0.12 & $4 \mathrm{KP}$ & ElimLin \\
\hline 64 & 4 & 192 & 148 & \multirow[t]{2}{*}{0.05} & \multirow{4}{*}{2.21} & $1 \mathrm{KP}$ & ElimLin \\
\hline 64 & 4 & 192 & 155 & & & $5 \mathrm{KP}$ & ElimLin \\
\hline 85 & 4 & 255 & 220 & 0.29 & & $1 \mathrm{KP}$ & ElimLin \\
\hline 85 & 4 & 255 & 215 & \multirow[t]{4}{*}{0.64} & & $1 \mathrm{KP}$ & ElimLin \\
\hline 85 & 4 & 255 & 220 & & 0.26 & $2 \mathrm{KP}$ & ElimLin \\
\hline 85 & 4 & 255 & 215 & & 0.90 & $3 \mathrm{KP}$ & ElimLin \\
\hline 85 & 4 & 255 & 210 & & 1.33 & $4 \mathrm{KP}$ & ElimLin \\
\hline 16 & 5 & 48 & 0 & \multirow[t]{2}{*}{3} & \multirow[b]{2}{*}{0.03} & $8 \mathrm{CP}$ & ElimLin \\
\hline 40 & 5 & 120 & 85 & & & $2 \mathrm{CP}$ & ElimLin \\
\hline 32 & 6 & 96 & 60 & \multirow{7}{*}{$\begin{array}{c}2.5 \\
1 \\
2.4 \\
3\end{array}$} & \multirow{7}{*}{$\begin{array}{c}3 \\
180.5 \\
4.5\end{array}$} & $16 \quad \mathrm{CP}$ & ElimLin \\
\hline 40 & 6 & 120 & 80 & & & $8 \mathrm{CP}$ & ElimLin \\
\hline 64 & 6 & 192 & 155 & & & $4 \mathrm{CP}$ & ElimLin \\
\hline 85 & 6 & 255 & 210 & & & $2 \mathrm{CP}$ & ElimLin \\
\hline 85 & 6 & 255 & 220 & & & $16 \mathrm{CP}$ & ElimLin \\
\hline 85 & 6 & 255 & 210 & & & $64 \mathrm{CP}$ & ElimLin \\
\hline 128 & 6 & 384 & 344 & & & $2 \mathrm{CP}$ & ElimLin \\
\hline
\end{tabular}

$B$ : Number of S-boxes per round. To obtain the block size, $B$ is multiplied by 3 .

$N_{r}$ : Number of rounds

$g$ : Number of guessed LSB of the key

Running Time ${ }^{1}$ : Running time until we achieve equations only in key variables (no other internal variables). When this is achieved, the cipher is close to be broken, but not yet (see Sec. 5.2).

Running Time ${ }^{2}$ : Attack running time for recovering $(\# k e y-g)$ bits of the key.

KP: Known plaintext

CP: Chosen plaintext

\subsection{Simulations on MIBS}

Similar to the LBlock block cipher, MIBS is also a lightweight Feistel-based block cipher, aimed at constrained environments, such as RFID tags and sensor networks 38 . It operates on 64-bit blocks, uses keys of 64 or 80 bits and iterates 32 rounds. For a detailed specification of the cipher, see 38.

Currently, the best cryptanalysis results is a linear attack reaching 18-round MIBS with data complexity $2^{61}$ and time complexity of $2^{76}[5]$. In fact, statistical attacks often require very large number of samples. This is not always achievable in practice. 
Table 2. Algebraic attack complexities on reduced-round LBlock using ElimLin and PolyBoRi

\begin{tabular}{|c|c|c|c|c|c|}
\hline$N_{r}$ & $\# k e y$ & $g$ & $\begin{array}{l}\text { Running Time } \\
\text { (in hours) }\end{array}$ & Data & $\begin{array}{l}\text { Attack } \\
\text { notes }\end{array}$ \\
\hline 8 & 80 & 32 & U & $6 \mathrm{KP}$ & Lin \\
\hline 8 & 80 & 32 & rashed & $6 \mathrm{KI}$ & PolyBol \\
\hline
\end{tabular}

$N_{r}$ : Number of rounds

$g$ : Number of guessed LSB of the key

KP: Known plaintext

CP: Chosen plaintext

We break 4 and 3 rounds of MIBS80 and MIBS64 using 32 and 2 samples deploying an ordinary PC by ElimLin. Our results are summarized in Table 3. In 2 out of 3 experiments, PolyBoRi crashes due to running out of memory. This is the first algebraic analysis of the cipher.

The designers in 38] have evaluated the security of their cipher with respect to algebraic attacks. They used the complexity of XSL algorithm for this evaluation, which is not a precise measurement for evaluating resistance of a cipher against algebraic attacks, since effectiveness of XSL is still controversial and under speculation. There are better methods such as SAT solvers [3] which solve MQ problem faster than expected due to the system being overdefined and sparse.

Let assume XSL can be precise enough to evaluate the security of a cipher with respect to algebraic attacks. According to [2038, the complexity of XSL can be evaluated with the work factor. For MIBS, work factor is computed as:

$$
\mathrm{WF}=\Gamma^{\omega}\left((\text { Block Size }) \cdot N_{r}^{2}\right)^{\omega\left\lceil\frac{T}{r}\right\rceil}
$$

where $\Gamma$ is a parameter which depends only on the S-box. For MIBS, $\Gamma=85.56$. The value $r=21$ is the number of equations the S-box can be represented with. $T=37$ is the number of monomials in that representation. $\omega=2.37$ is the exponent of the Gaussian elimination complexity. The work factor for attacking 5-round MIBS is $\mathrm{WF}=2^{65.65}$ which is worse than an exhaustive key search for MIBS64. Deploying SAT solving techniques using MiniSAT 2.0 28, we can break 5 rounds of MIBS64 (see Table 3). Our strategy is exactly the same as [3]. Table 3already shows that we can do better than $2^{65.65}$ for MIBS64. We can perform a very similar attack on MIBS80. This already shows that considering the complexity of XSL is not a precise measure to evaluate the security of a cipher against algebraic cryptanalysis. Complexity of attacking such system with XL is extremely high.

We believe that due to the similarity between the structure of MIBS and LBlock, we can compare them with respect to algebraic attacks. As can be seen from the table of attacks, LBlock is much weaker. This is not surprising though, since the linear layer of LBlock is much weaker than MIBS, since it is nibblewise instead of bit-wise. So, we could attack twice more rounds of LBlock. Thus, although LBlock is lighter with respect to the number of gates, but it provides a lower level of security with respect to algebraic attacks. 
Table 3. Algebraic attack complexities on reduced-round MIBS using ElimLin, PolyBoRi and MiniSAT 2.0

\begin{tabular}{c|c|c|c|cc|c}
\hline$N_{r}$ & \#key & $g$ & $\begin{array}{c}\text { Running Time } \\
\text { (in hours) }\end{array}$ & Data & $\begin{array}{c}\text { Attack } \\
\text { notes }\end{array}$ \\
\hline 4 & 80 & 20 & 0.137 & 32 & $\mathrm{KP}$ & ElimLin \\
4 & 80 & 20 & crashed & 32 & $\mathrm{KP}$ & PolyBoRi \\
\hline 5 & 64 & 16 & 0.395 & 6 & $\mathrm{KP}$ & MiniSAT 2.0 \\
5 & 64 & 16 & crashed & 6 & $\mathrm{KP}$ & PolyBoRi \\
3 & 64 & 0 & 0.006 & 2 & $\mathrm{KP}$ & ElimLin \\
3 & 64 & 0 & 0.002 & 2 & $\mathrm{KP}$ & PolyBoRi \\
\hline
\end{tabular}

$N_{r}$ : Number of rounds

$g$ : Number of guessed LSB of the key

KP: Known plaintext

CP: Chosen plaintext

\section{A Comparison between ElimLin and PolyBoRi}

Gröbner basis is currently one of the most successful methods for solving polynomial systems of equations. However, it has its own restrictions. The main bottleneck of the Gröbner basis techniques is the memory requirement and therefore most of the Gröbner basis attacks use relatively small number of samples. It is worthwhile to mention that ElimLin is a subroutine is Gröbner basis computations. But, ElimLin algorithm as a single tool requires a large number of samples to work.

The Gröbner basis solve the system by reductions according to a pre-selected ordering, which can lead to high degree dense polynomials. ElimLin uses the fact that multiple samples provide an additional information to the solver, and therefore the key might be found even if when we restrict the reduction to degree 2 .

Next, we compare the current state of the art implementation of F4 algorithm PolyBoRi and our implementation of ElimLin. In the cases where ElimLin behaves better than PolyBoRi, it does not mean that ElimLin is superior to F4 algorithm. In fact, it just means that there exists a better implementation for ElimLin than for F4 for some particular systems of equations. F4 uses a fixed ordering for monomials and therefore it does not preserve the sparsity in its intermediate steps. On the other hand, our implementation of ElimLin performs several sparsity preserving techniques by changing the ordering. This drops the total number of monomials and makes it memory efficient.

Table 2 and Table 3 show that PolyBoRi requires too much memory and crashes for a large number of samples. At the same time, our implementation of ElimLin is slightly slower than PolyBoRi implementation attacking 2 samples of 3-round MIBS64 as in Table3. This demonstrates that our implementation of ElimLin can be more effective than PolyBoRi and vice versa, depending on memory requirements of PolyBoRi. However, whenever the system is solvable by our implementation of ElimLin, our experiments revealed that PolyBoRi does not give a significant advantage over ElimLin because the memory requirements are too high. 
While PolyBoRi may yield a solution for a few samples, the success of ElimLin is determined by the number of samples provided to the algorithm. The evaluation of the number of sufficient samples in ElimLin is still an open problem.

We see that often preserving the degree by simple linear algebra techniques can outperform the more sophisticated Gröbner basis algorithms, mainly due to the structural properties of the system of equations of a cryptographic primitive (such as sparsity). ElimLin takes advantage of such structural properties and uncovers some hidden linear equations using multiple samples. According to our experiments, PolyBoRi does not seem to be able to take advantage of these structural properties as would be expected which results in higher memory requirements than would be necessary and ultimately their failure for large systems, even though it is clearly possible for the algorithm to find the solution in reasonable time. Finally, we need more efficient implementations and data structures for both ElimLin and Gröbner basis algorithms.

\section{Further Work and Some Conjectures}

An interesting area of research is to estimate the number of linear equations in ElimLin or anticipate how this number evolves in the succeeding iterations or evaluate after how many iterations ElimLin finishes. Also, to anticipate how many samples is enough to make the system collapse by ElimLin. Last but not least, it is prominent to find a very efficient method for implementing ElimLin and to find the most appropriate data structure to choose.

There are some evidence which illustrate that ElimLin does not reveal all hidden linear equations in the structure of the cipher up to a specific degree. We give an example, demonstrating such an evidence:

Assume there exists an equation in the system which can be represented as $\ell(x) g(x)+1=0$ over $\mathrm{GF}(2)$, where $\ell(x)$ is a polynomial of degree one and $g(x)$ is a polynomial of degree at most $d-1$. Running ElimLin on this single equation trivially fails. But, if we multiply both sides of the equation by $\ell(x)$, we obtain $\ell(x) g(x)+\ell(x)=0$. Summing these two equations, we derive $\ell(x)=1$. This hidden linear equation can be simply captured by the XL algorithm, but can not be captured by ElimLin. There exist multiple other examples which demonstrate that ElimLin does not generate all the hidden linear equations. To generate all such linear equations, the degree-bounded Gröbner basis can be used.

For big ciphers, for example the full AES, it is also plausible that:

Conjecture 1. For each number of rounds $X$, there exists $Y$ such that AES is broken by ElimLin given $Y$ Chosen or Known Plaintext-Ciphertext pairs.

Disproving the above conjecture leads to the statement that "AES can not be broken by algebraic attack at degree 2". But maybe this conjecture is true, then the capacities of the ElimLin attack are considerable and it works for any number of rounds $X$. As a consequence, if for $X=14$ this $Y$ is not too large, say less than $2^{64}$, the AES-256 will be broken faster than brute force by ElimLin at degree 
2, which is much simpler than Gröbner basis objective of breaking it at degree 3 or 4 with $1 \mathrm{KP}$.

ElimLin is a polynomial time algorithm. If it can be shown that a polynomial number of samples is enough to gain a high success rate for ElimLin, this can already be considered a breakthrough in cryptography. Unfortunately, the correctness of this statement is not clear.

\section{Conclusion}

In this paper, we proved that ElimLin can be formulated in terms of a sequence of intersections of vector spaces. We showed that different monomial orderings and any affine bijective variable change do not influence the result of the algorithm. We did some predictions on the evolution of linear equations in the succeeding iterations in ElimLin. We presented multiple attacks deploying ElimLin against CTC2, LBlock and MIBS block ciphers.

\section{References}

1. Armknecht, F., Ars, G.: Algebraic Attacks on Stream Ciphers with Gröbner Bases. In: Gröbner Bases, Coding, and Cryptography, pp. 329-348 (2009)

2. Aumasson, J.-P., Henzen, L., Meier, W., Naya-Plasencia, M.: QuARK: A Lightweight Hash. In: Mangard, S., Standaert, F.-X. (eds.) CHES 2010. LNCS, vol. 6225, pp. 1-15. Springer, Heidelberg (2010)

3. Bard, G., Courtois, N., Jefferson, C.: Efficient Methods for Conversion and Solution of Sparse Systems of Low-Degree Multivariate Polynomials over GF(2) via SATSolvers. Presented at ECRYPT Workshop Tools for Cryptanalysis (2007), http://eprint.iacr.org/2007/024.pdf

4. Bard, G.V.: Algebraic Cryptanalysis. Springer (2009)

5. Bay, A., Nakahara Jr., J., Vaudenay, S.: Cryptanalysis of Reduced-Round MIBS Block Cipher. In: Heng, S.-H., Wright, R.N., Goi, B.-M. (eds.) CANS 2010. LNCS, vol. 6467, pp. 1-19. Springer, Heidelberg (2010)

6. Bogdanov, A., Knežević, M., Leander, G., Toz, D., Varıcı, K., Verbauwhede, I.: SPOngent: A Lightweight Hash Function. In: Preneel, B., Takagi, T. (eds.) CHES 2011. LNCS, vol. 6917, pp. 312-325. Springer, Heidelberg (2011)

7. Bogdanov, A., Knudsen, L.R., Leander, G., Paar, C., Poschmann, A., Robshaw, M., Seurin, Y., Vikkelsoe, C.: PRESENT: An Ultra-Lightweight Block Cipher. In: Paillier, P., Verbauwhede, I. (eds.) CHES 2007. LNCS, vol. 4727, pp. 450-466. Springer, Heidelberg (2007)

8. Brickenstein, M., Dreyer, A.: PolyBoRi: A framework for Gröbner basis computations with Boolean polynomials. In: Electronic Proceedings of MEGA 2007 (2007), http://www.ricam.oeaw.ac.at/mega2007/electronic/26.pdf

9. Buchberger, B.: Bruno Buchberger's PhD thesis 1965: An algorithm for finding the basis elements of the residue class ring of a zero dimensional polynomial ideal. Journal of Symbolic Computation 41(3-4), 475-511 (2006)

10. Courtois, N.T.: Higher Order Correlation Attacks, XL Algorithm and Cryptanalysis of Toyocrypt. In: Lee, P.J., Lim, C.H. (eds.) ICISC 2002. LNCS, vol. 2587, pp. 182-199. Springer, Heidelberg (2003) 
11. Courtois, N.T.: Fast Algebraic Attacks on Stream Ciphers with Linear Feedback. In: Boneh, D. (ed.) CRYPTO 2003. LNCS, vol. 2729, pp. 176-194. Springer, Heidelberg (2003)

12. Courtois, N.: CTC2 and Fast Algebraic Attacks on Block Ciphers Revisited. In: Cryptology ePrint Archive (2007), http://eprint.iacr.org/2007/152.pdf

13. Courtois, N.: How Fast can be Algebraic Attacks on Block Ciphers? In: Symmetric Cryptography. Dagstuhl Seminar Proceedings, vol. 07021 (2007)

14. Courtois, N.: The Dark Side of Security by Obscurity - and Cloning MiFare Classic Rail and Building Passes, Anywhere, Anytime. In: SECRYPT, pp. 331-338 (2009)

15. Courtois, N.: Algebraic Complexity Reduction and Cryptanalysis of GOST. In: Cryptology ePrint Archive (2011), http://eprint.iacr.org/2011/626

16. Courtois, N.T., Bard, G.V.: Algebraic Cryptanalysis of the Data Encryption Standard. In: Galbraith, S.D. (ed.) Cryptography and Coding 2007. LNCS, vol. 4887, pp. 152-169. Springer, Heidelberg (2007)

17. Courtois, N.T., Debraize, B.: Algebraic Description and Simultaneous Linear Approximations of Addition in Snow 2.0. In: Chen, L., Ryan, M.D., Wang, G. (eds.) ICICS 2008. LNCS, vol. 5308, pp. 328-344. Springer, Heidelberg (2008)

18. Courtois, N.T., Meier, W.: Algebraic Attacks on Stream Ciphers with Linear Feedback. In: Biham, E. (ed.) EUROCRYPT 2003. LNCS, vol. 2656, pp. 345-359. Springer, Heidelberg (2003)

19. Courtois, N.T., O'Neil, S., Quisquater, J.-J.: Practical Algebraic Attacks on the Hitag2 Stream Cipher. In: Samarati, P., Yung, M., Martinelli, F., Ardagna, C.A. (eds.) ISC 2009. LNCS, vol. 5735, pp. 167-176. Springer, Heidelberg (2009)

20. Courtois, N.T., Pieprzyk, J.: Cryptanalysis of Block Ciphers with Overdefined Systems of Equations. In: Zheng, Y. (ed.) ASIACRYPT 2002. LNCS, vol. 2501, pp. 267-287. Springer, Heidelberg (2002)

21. Courtois, N.T., Klimov, A.B., Patarin, J., Shamir, A.: Efficient Algorithms for Solving Overdefined Systems of Multivariate Polynomial Equations. In: Preneel, B. (ed.) EUROCRYPT 2000. LNCS, vol. 1807, pp. 392-407. Springer, Heidelberg (2000)

22. De Cannière, C., Dunkelman, O., Knežević, M.: KATAN and KTANTAN - A Family of Small and Efficient Hardware-Oriented Block Ciphers. In: Clavier, C., Gaj, K. (eds.) CHES 2009. LNCS, vol. 5747, pp. 272-288. Springer, Heidelberg (2009)

23. Dinur, I., Shamir, A.: Cube Attacks on Tweakable Black Box Polynomials. In: Joux, A. (ed.) EUROCRYPT 2009. LNCS, vol. 5479, pp. 278-299. Springer, Heidelberg (2009)

24. Dinur, I., Shamir, A.: Breaking Grain-128 with Dynamic Cube Attacks. In: Joux, A. (ed.) FSE 2011. LNCS, vol. 6733, pp. 167-187. Springer, Heidelberg (2011)

25. Dunkelman, O., Keller, N.: Linear Cryptanalysis of CTC. In: Cryptology ePrint Archive (2006), http://eprint.iacr.org/2006/250.pdf

26. Dunkelman, O., Keller, N.: Cryptanalysis of CTC2. In: Fischlin, M. (ed.) CT-RSA 2009. LNCS, vol. 5473, pp. 226-239. Springer, Heidelberg (2009)

27. Eén, N., Sörensson, N.: MiniSat 2.0. An open-source SAT solver package, http://www.cs.chalmers.se/Cs/Research/FormalMethods/MiniSat/

28. Een, N., Sorensson, N.: Minisat - A SAT Solver with Conflict-Clause Minimization. In: Theory and Applications of Satisfiability Testing (2005)

29. Engels, D., Saarinen, M.-J.O., Schweitzer, P., Smith, E.M.: The Hummingbird-2 Lightweight Authenticated Encryption Algorithm. In: Juels, A., Paar, C. (eds.) RFIDSec 2011. LNCS, vol. 7055, pp. 19-31. Springer, Heidelberg (2012) 
30. Faugère, J.: A new effcient algorithm for computing Gröbner bases (F4). Journal of Pure and Applied Algebra 139(1-3), 61-88 (1999)

31. Faugère, J.: A new efficient algorithm for computing Gröbner bases without reduction to zero (F5). In: Symbolic and Algebraic Computation - ISSAC, pp. 75-83 (2002)

32. Fusco, G., Bach, E.: Phase transition of multivariate polynomial systems. Journal of Mathematical Structures in Computer Science 19(1) (2009)

33. Ghasemzadeh, M.: A New Algorithm for the Quantified Satisfiability Problem, Based on Zero-suppressed Binary Decision Diagrams and Memoization. PhD thesis, University of Potsdam, Germany (2005)

34. Gong, Z., Nikova, S., Law, Y.W.: KLEIN: A New Family of Lightweight Block Ciphers. In: Juels, A., Paar, C. (eds.) RFIDSec 2011. LNCS, vol. 7055, pp. 1-18. Springer, Heidelberg (2012)

35. Guo, J., Peyrin, T., Poschmann, A.: The PHOTON Family of Lightweight Hash Functions. In: Rogaway, P. (ed.) CRYPTO 2011. LNCS, vol. 6841, pp. 222-239. Springer, Heidelberg (2011)

36. Guo, J., Peyrin, T., Poschmann, A., Robshaw, M.J.B.: The LED Block Cipher. In: Preneel, B., Takagi, T. (eds.) CHES 2011. LNCS, vol. 6917, pp. 326-341. Springer, Heidelberg (2011)

37. Indesteege, S., Keller, N., Dunkelman, O., Biham, E., Preneel, B.: A Practical Attack on KeeLoq. In: Smart, N.P. (ed.) EUROCRYPT 2008. LNCS, vol. 4965, pp. 1-18. Springer, Heidelberg (2008)

38. Izadi, M., Sadeghiyan, B., Sadeghian, S., Arabnezhad, H.: MIBS: A New Lightweight Block Cipher. In: Garay, J.A., Miyaji, A., Otsuka, A. (eds.) CANS 2009. LNCS, vol. 5888, pp. 334-348. Springer, Heidelberg (2009)

39. Knudsen, L., Leander, G., Poschmann, A., Robshaw, M.J.B.: PRINTCIPher: A Block Cipher for IC-Printing. In: Mangard, S., Standaert, F.-X. (eds.) CHES 2010. LNCS, vol. 6225, pp. 16-32. Springer, Heidelberg (2010)

40. Magma, software package, http://magma.maths.usyd.edu.au/magma/

41. Mroczkowski, P., Szmidt, J.: The Cube Attack on Courtois Toy Cipher. In: Cryptology ePrint Archive (2009), http://eprint.iacr.org/2009/497.pdf

42. Murphy, S., Robshaw, M.J.B.: Essential Algebraic Structure within the AES. In: Yung, M. (ed.) CRYPTO 2002. LNCS, vol. 2442, pp. 1-16. Springer, Heidelberg (2002)

43. Nakahara Jr., J., Sepehrdad, P., Zhang, B., Wang, M.: Linear (Hull) and Algebraic Cryptanalysis of the Block Cipher PRESENT. In: Garay, J.A., Miyaji, A., Otsuka, A. (eds.) CANS 2009. LNCS, vol. 5888, pp. 58-75. Springer, Heidelberg (2009)

44. Raddum, H., Semaev, I.: Solving Multiple Right Hand Sides linear equations. Journal of Designs, Codes and Cryptography 49(1-3), 147-160 (2008)

45. Shannon, C.E.: Communication theory of secrecy systems. Bell System Technical Journal 28 (1949)

46. Shibutani, K., Isobe, T., Hiwatari, H., Mitsuda, A., Akishita, T., Shirai, T.: Piccolo: An Ultra-Lightweight Blockcipher. In: Preneel, B., Takagi, T. (eds.) CHES 2011. LNCS, vol. 6917, pp. 342-357. Springer, Heidelberg (2011)

47. Vielhaber, M.: Breaking ONE.FIVIUM by AIDA an Algebraic IV Differential Attack. In: Cryptology ePrint Archive (2007), http://eprint.iacr.org/2007/413

48. Weinmann, R.: Evaluating Algebraic Attacks on the AES. Master's thesis, Technische Universität Darmstadt (2003)

49. Wu, W., Zhang, L.: LBlock: A Lightweight Block Cipher. In: Lopez, J., Tsudik, G. (eds.) ACNS 2011. LNCS, vol. 6715, pp. 327-344. Springer, Heidelberg (2011) 